\title{
VÉGÉTATION AQUATIQUE ET PEUPLEMENT PISCIAIRE : APPROCHE EXPÉRIMENTALE DE L'ENLÈVEMENT DES MACROPHYTES DANS LES RADIERS D'UN COURS D'EAU BRETON.
}

\author{
J.M. ROUSSEL (1), A. BARDONNET (1), J. HAURY (1, 2), J.L. BAGLINIÈRE (1) \\ et E. PRÉVOST (1)
}

(1) Institut National de la Recherche Agronomique, Laboratoire d'Écologie Aquatique, 65 rue de Saint-Brieuc, 35042 Rennes Cedex, France.

(2) Ecole Nationale Supérieure Agronomique de Rennes, Laboratoire d'Écologie et Sciences Phytosanitaires, 65 rue de Saint-Brieuc, 35042 Rennes Cedex, France.

\section{RÉSUMÉ}

L'influence du recouvrement végétal des radiers d'une rivière à saumon de Bretagne (le Scorff) sur les préférences d'habitat de son peuplement pisciaire a été étudiée durant deux années consécutives. Sur deux radiers de 600 et $800 \mathrm{~m}^{2}$ fortement végétalisés (plus de $70 \%$ de recouvrement), l'expérimentation in situ a consisté à enlever les macrophytes sur la moitié de la surface de chaque radier (du centre du chenal jusqu'à la rive). Les conséquences mésologiques de l'enlèvement des végétaux ont surtout consisté en une forte augmentation des vitesses de courant ( $60 \%$ en moyenne) dans les habitats où les végétaux avaient été arrachés. Les résultats des pêches électriques réalisées un mois après l'enlèvement des macrophytes (mois de juin) ont mis en évidence des biomasses et des densités plus faibles dans les habitats sans végétaux. Les mêmes espèces étaient présentes dans les deux types d'habitat, mais les juvéniles de saumon atlantique (Salmo salar L.) étaient en moyenne deux fois plus nombreux dans les habitats sans végétaux (30 ind.100 $\mathrm{m}^{-2}$ ) qu'avec végétaux (15 ind. $100 \mathrm{~m}^{-2}$ ). Par contre, les densités de loches (Barbatula barbatula L.) et de vairons (Phoxinus phoxinus L.) étaient en moyenne de 47 ind. $100 \mathrm{~m}^{-2}$ dans les habitats végétalisés, c'est-à-dire 2 à 4 fois plus fortes que dans les habitats sans végétaux. Les résultats indiquent que le développement saisonnier des macrophytes est un important facteur structurant de l'habitat pisciaire dans les rivières du Massif Armoricain et suggèrent une diminution de la capacité d'accueil des radiers en juvéniles de saumon au printemps lors de la pousse des végétaux.

Mots-clés : renoncules aquatiques, poisson, préférence d'habitat, faciès d'écoulement, Salmo salar, capacité d'accueil.

\section{AQUATIC PLANT AND FISH ASSEMBLAGE : A MACROPHYTE REMOVAL EXPERIMENT IN STREAM RIFFLE HABITATS IN A LOWLAND SALMONID RIVER (BRITTANY, FRANCE).}

\section{ABSTRACT}

The effect of aquatic weed development on fish habitat preferences was studied during a two-year in situ experiment in lotic habitats of the Scorff river (Brittany, France). In 
the two riffles studied ( 800 and $600 \mathrm{~m}^{2}$ ), macrophytes (essentially Ranunculus penicillatus) covered more than $70 \%$ of the total surface area during the growing season in spring. In May, macrophytes were removed from one stream bank to the center of the riverbed (in each riffle) in order to create habitat without aquatic vegetation. The main effect of macrophyte removal on the physical habitat variables was an increase in water velocity $(60 \%$ on average). One month later, electrofishing surveys showed greater fish biomass and higher densities in vegetated habitats. The same species were present in both habitat types but density in Atlantic salmon parr (Salmo salar L.) was higher in habitats without macrophytes (30 ind.100 m-2 on average) than in vegetated habitats (15 ind.100 $\mathrm{m}^{-2}$ on average). Conversely, stone loach (Barbatula barbatula L.) and European minnow (Phoxinus phoxinus L.) densities were 47 ind. $100 \mathrm{~m}^{-2}$ on average in vegetated habitats, i.e. two to four times greater than in habitats without vegetation. The results point out that macrophytes can change drastically habitat conditions and suggest a decrease in the carrying capacity of riffles for age-0 salmon parr.

Key-words : macrophyte, fish community, habitat partitioning, geomorphological unit, Salmo salar, carrying capacity.

\section{INTRODUCTION}

La présence des végétaux aquatiques est un élément caractéristique des rivières du Massif Armoricain dont le développement est probablement favorisé par l'anthropisation des milieux. En effet, l'augmentation des apports en nutriments liée à des pollutions organiques ponctuelles (DANIEL et HAURY, 1995) ainsi que les altérations de la ripisylve (CHAMPIGNEULLE, 1978 ; DAWSON et KERN-HANSEN, 1979 ; DAWSON et HASLAM, 1983) sont de nature à renforcer les conditions favorables au développement des végétaux aquatiques. Parmi les macrophytes à forte capacité de recouvrement, les renoncules aquatiques (Ranunculus penicillatus) se développent au printemps essentiellement sur les plats courants et les radiers où elles peuvent facilement occuper plus de la moitié de la surface mouiliée (HAURY et BAGLINIĖRE, 1996). Dans les rivières de faible pente $(<2 \%$ ), les radiers sont des unités d'écoulement typiques de la géomorphologie du chenal (BISSON et MONTGOMERY, 1996). Leur rôle dans l'écologie du peuplement pisciaire est multiple. D'un point de vue trophique, its représentent des zones à forte production de larves d'invertébrés (LOGAN et BROOKER, 1983 ; BROWN et BRUSSOCK, 1991) dont se nourrissent de nombreux poissons. Ils constituent, de plus, l'essentiel des zones de nurserie des juvéniles de saumon atlantique (Salmo salar) et jouent ainsi un rôle primordial dans la production en juvéniles de saumon (BAGLINIĖRE et CHAMPIGNEULLE, 1986).

Le développement saisonnier des macrophytes peut entraîner d'importantes modifications de l'habitat, notamment par ralentissement des vitesses de courant et piégeage de sédiments (SAND-JENSEN et al., 1989 ; DAWSON et CHARLTON, 1988 in VINSON et al., 1992 ; HAURY et BAGLINIĖRE, 1996). En changeant l'hydraulique et le substrat, les macrophytes sont donc susceptibles de modifier les conditions d'habitat pour le poisson et ainsi de changer la capacité d'accueil du site (HEARNE et al., 1994). Cependant, l'influence des macrophytes sur les préférences d'habitat des poissons en rivière reste mal connue. Une approche expérimentale in situ par enlèvement des renoncules a donc été mise en place sur des radiers du cours principal du Scorft, rivière à saumon du Massif Armoricain. Les objectifs de l'étude sont de caractériser les modifications mésologiques engendrées par l'enlèvement des macrophytes et d'en évaluer l'impact en termes de choix d'habitat pour les juvéniles de saumon et les autres espèces de la communauté pisciaire. 


\section{SITE D'ÉTUDE}

L'étude s'est déroulée en 1996 et 1997 sur le Scorff (Figure 1), petit cours d'eau de la Bretagne Sud, d'une longueur de $75 \mathrm{~km}$, qui s'écoule sur un substrat de type granitique et schisteux. Le bassin versant est de $480 \mathrm{~km}^{2}$ et le profil en long est caractérisé par une pente irrégulière, variant de $0,015 \%$ à $2 \%$ (CHAMPIGNEULLE, 1978). Pendant la période d'étude, la température de l'eau enregistrée sur la partie aval de la rivière (Pont-Scorff, Figure 1) a varié de $0^{\circ} \mathrm{C}$ (moyenne journalière la plus basse enregistrée au mois de janvier 1997) à $22^{\circ} \mathrm{C}$ (moyenne journalière la plus haute au mois de juillet 1996). Les chroniques de débit enregistrées à Pont-Kerlo (Figure 1) indiquent que pendant la période estivale de basses eaux le débit descend aux alentours de $1 \mathrm{~m}^{3} \cdot \mathrm{s}^{-1}$ pour atteindre des moyennes mensuelles de 6 à $10 \mathrm{~m}^{3} . \mathrm{s}^{-1}$ pendant les périodes de hautes eaux hivernales. Au printemps, un développement important des macrophytes, concernant essentiellement la renoncule en pinceau (Ranunculus penicillatus), est observé sur les radiers du cours moyen du Scorff (DANIEL et HAURY, 1995).

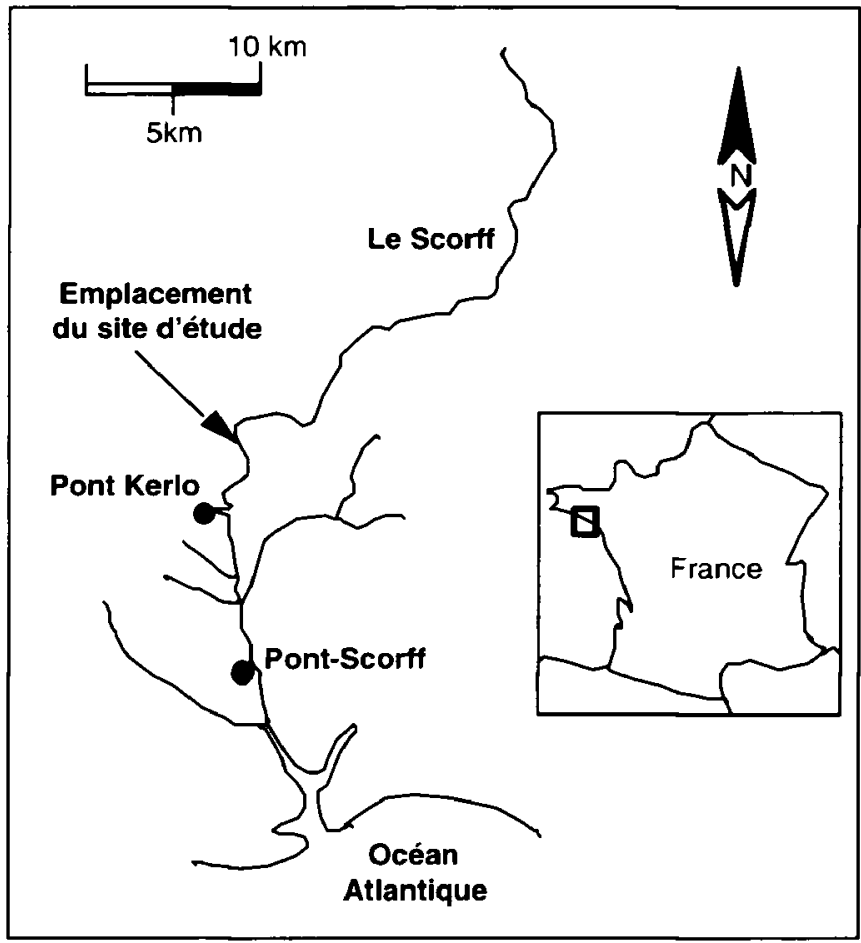

Figure 1

Le bassin du Scorff et emplacement du site d'étude.

\section{Figure 1}

\section{The Scorff river and the location of the study site.}

\section{MATÉRIEL ET MÉTHODES}

Deux radiers de la partie médiane du cours d'eau (Figure 1), distants d'environ $300 \mathrm{~m}$ l'un de l'autre et occupant toute la largeur du Scorff (soit 25 à $30 \mathrm{~m}$ ), ont été choisis pour l'étude. Ils représentent des surfaces de $600 \mathrm{~m}^{2}$ (radier amont) et $800 \mathrm{~m}^{2}$ (radier aval). Préalablement à toute intervention sur la végétation aquatique, une caractérisation globale de l'habitat par transect est réalisée chaque année au mois de mai, à raison d'un transect tous les 5 mètres (soit 6 transects sur le radier aval et 5 transects sur le radier amont) et un point de mesure (ou point-contact) tous les mètres le long des transects. A chaque point-contact, 
les mesures suivantes sont réalisées : profondeur $( \pm 1 \mathrm{~cm})$, vitesse de courant au tiers inférieur de la colonne d'eau $\left( \pm 1 \mathrm{~cm} \cdot \mathrm{s}^{-1}\right.$, mesures par courantomètre électromagnétique), granulométrie (en 1996 uniquement, estimation visuelle selon la classification de CAILLEUX (1954, Tableau I)). Les pourcentages de recouvrement sont estimés pour les trois classes majoritaires sur une surface de $200 \mathrm{~cm}^{2}$ à chaque point-contact (total des trois classes dominantes $=100 \%$ ). Un complément de mesure est également réalisé sur trois des transects de chaque radier : vitesses de courant au fond et à la surface de la lame d'eau, recouvrement en renoncules d'un point-contact à l'autre le long du transect $( \pm 5 \mathrm{~cm})$, hauteurs d'eau vacantes en dessous et au-dessus de la touffe de renoncule (uniquement en 1997).

Immédiatement après cette première série de mesures d'habitat, les renoncules sont enlevées à la main sur la moitié de chaque radier, créant ainsi deux types d'habitat ou demiradiers avec et sans végétaux (Figure 2). Afin d'éviter un biais d'échantillonnage dû à l'hétérogénéité transversale du lit (faible sur le radier aval mais plus importante sur le radier amont), les côtés expérimentaux (végétaux enlevés) et les côtés intacts (végétaux laissés en place) ont été inversés d'une année sur l'autre. Le jour suivant l'enlèvement des renoncules, une seconde série de mesures mésologiques est réalisée pour évaluer les modifications hydrauliques. Trois transects par radier (situés rigoureusement sur les mêmes emplacements qu'avant l'enlèvement des renoncules) sont alors réalisés, en mesurant à chaque point-contact la profondeur, les vitesses de courant au fond, au tiers et à la surface de la colonne d'eau et la granulométrie (en 1996 uniquement).
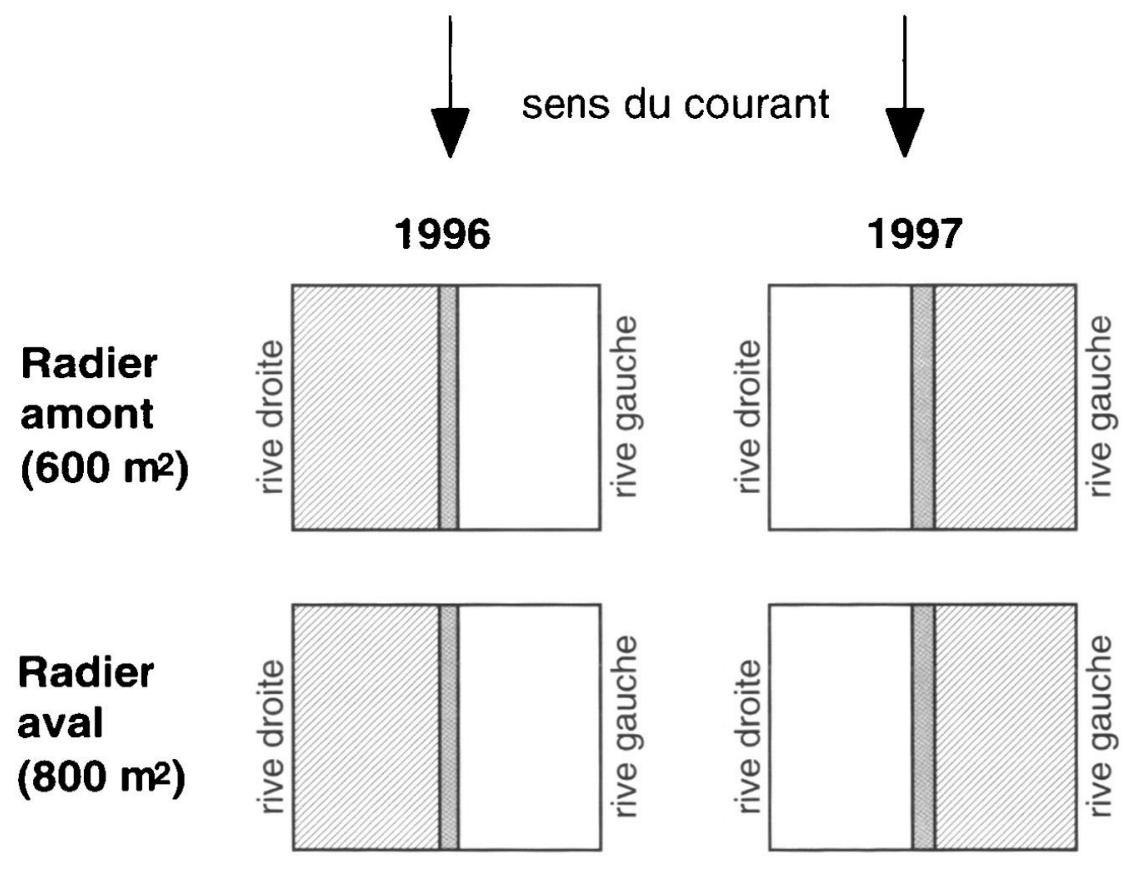

\section{Figure 2}

Schéma de l'expérimentation réalisée sur les deux radiers au cours des deux années d'étude. Zone blanche : habitat expérimental (végétation enlevée). Zone hachurée : habitat intact (végétation laissée). Zone grise : zone tampon.

\section{Figure 2}

Design of the experiment performed in the upstream and the downstream riffle in 1996 and 1997. White area : experimental habitat (vegetation removed). Hatched area : intact habitat (vegetation left). Grey area : buffer zone. 
Un mois après l'enlèvement des renoncules, deux journées d'inventaire du peuplement pisciaire sont réalisées chaque année, le radier aval étant pêché le premier jour et l'habitat sans végétaux étant systématiquement pêché avant l'habitat végétalisé. La méthode utilisée est celle de la pêche électrique par enlèvements successifs (courant continu, voltage 200 à $250 \mathrm{~V}$, intensité 0,75 à $1,5 \mathrm{~A}$ ). Trois porteurs d'anode (30 cm de diamètre) progressent côte à côte dans le demi-radier pêché, de l'aval vers l'amont. Chacun d'entre eux est accompagné par deux porteurs d'épuisettes. Le premier est équipé d'une épuisette semi-circulaire à fond plat de $60 \mathrm{~cm}$ de diamètre (maille $3 \mathrm{~mm}$ ) plaquée sur le fond du radier et d'une épuisette circulaire plus mobile de $20 \mathrm{~cm}$ de diamètre (maille $3 \mathrm{~mm}$ ), le second ne porte qu'une épuisette circulaire de $20 \mathrm{~cm}$ de diamètre (maille $3 \mathrm{~mm}$ ). Après récupération, les poissons sont mesurés $( \pm 1 \mathrm{~mm})$, pesés $( \pm 0,1 \mathrm{~g}$ ) et stockés en viviers pendant la durée de la pêche. Dans les habitats sans végétaux, deux ou trois passages (selon l'efficacité de pêche) sont successivement effectués. Dans les habitats végétalisés, deux passages sont réalisés avec la végétation en place, puis un passage de récupération finale est fait après enlèvement total des renoncules. Les poissons capturés aux limites amont et aval des secteurs (bande de sécurité de 2 mètres de large), ainsi que dans une zone tampon de $4 \mathrm{~m}$ entre les deux habitats (avec et sans renoncules, Figure 2), sont comptabilisés à part.

\section{TRAITEMENT DES DONNÉES}

Chaque année, la comparaison des caractéristiques physiques des habitats pisciaires est effectuée en deux étapes. Dans un premier temps, les caractéristiques moyennes de chaque demi-radier avant l'enlèvement des renoncules sont calculées pour la profondeur, la vitesse au tiers de la colonne d'eau, la granulométrie, le recouvrement en renoncules (en pourcentage du linéaire) et le biovolume végétal (ou proportion du volume total d'eau occupé par les renoncules). Les différences entre demi-radiers appartenant au même faciès sont testées par des comparaisons de moyennes deux à deux (test $t$ ). Dans un second temps, les modifications d'hydraulique consécutives à l'enlèvement des renoncules sont évaluées par comparaison des distributions des profondeurs, des vitesses (correspondant à la moyenne des vitesses au fond, au tiers et en surface) et de la granulométrie avant et après enlèvement des renoncules. Sur chaque demi-radier, les profondeurs et vitesses moyennes sont découpées en classes de $5 \mathrm{~cm}$ et $10 \mathrm{~cm} . \mathrm{S}^{-1}$. Pour un même demi-radier (expérimental ou intact), la différence de fréquence classe à classe avant et après enlèvement des renoncules $\left(D F_{x}\right)$ est calculée selon la formule : $D F_{x}=F_{x}$ ' $-F_{x}$, où $F_{x}=$ la fréquence de la classe $X$ avant enlèvement des renoncules et $F_{x}$, la fréquence de cette même classe après enlèvement des renoncules. Lorsque $D F_{x}$ est égale à zéro, la fréquence de la classe $X$ de la variable considérée n'a pas varié après enlèvement des renoncules. Si $D F_{x}$ est différent de zéro, la fréquence de la classe $X$ augmente $\left(D F_{x}>0\right)$ ou diminue $\left(D F_{x}<0\right)$ après enlèvement des renoncules. Les modifications globales de l'hydraulique consécutives à l'enlèvement des renoncules sont ensuite testées pour chaque variable en comparant les distributions des fréquences cumulées avant et après enlèvement, grâce à des tests de rangs appariés (test de Wilcoxon).

Les poissons capturés dans les zones tampons (représentant en moyenne $8 \%$ des captures totales) ainsi que les surfaces de radier correspondantes (représentant $14 \%$ de la surface pêchée) sont retirés des calculs de densités. L'estimation des densités par espèce en habitat non-végétalisé est faite selon la méthode du maximum de vraisemblance proposée par ZIPPIN (1956) à l'aide du logiciel MicroFish 3.0 (VAN DEVENTER et PLATTS, 1989). Les intervalles de confiance à $95 \%$ autour de l'estimation sont calculés en référence à une distribution $t$ de Student. Cette méthode, pour laquelle la probabilité de capture est supposée constante d'un passage à l'autre, n'est pas applicable dans le cas des habitats végétalisés, puisque l'enlèvement des renoncules entre le deuxième et le troisième passages entraîne une modification des conditions de pêche. Dans ce cas, l'estimation de la densité la plus probable pour chaque espèce est calculée selon le schéma suivant : soient 
$\mathrm{C} 1, \mathrm{C} 2$ et $\mathrm{C} 3$ les effectifs de poissons pêchés respectivement lors des premier, deuxième et troisième passages. L'effectif restant après l'enlèvement des renoncules (C'3) est estimé en s'inspirant de la méthode Bayesienne proposée par CARLE et STRUB (1978). On calcule la distribution de probabilités des effectifs a posteriori connaissant $\mathrm{C} 3$ et en prenant pour distribution a priori de la probabilité de capture du troisième passage une loi uniforme, bornée par les efficacités de pêche maximum et minimum observées pour l'espèce considérée en habitats non-végétalisés. L'estimateur de $\mathrm{C}^{\prime} 3$ est alors la valeur la plus probable dans la distribution a posteriori. La densité estimée en habitat végétalisé $(\tilde{\mathrm{N}})$ correspond à la somme : $\tilde{\mathrm{N}}=\mathrm{C} 1+\mathrm{C} 2+\mathrm{C}^{\prime} 3$. L'intervalle a posteriori à $95 \%$ autour de $\tilde{\mathrm{N}}$ est déduit de la distribution de probabilités autour de C’3.

Les différences de densité pour chaque espèce dans les habitats végétalisés et nonvégétalisés sont évaluées par des tests de Wilcoxon, en appariant les densités estimées des demi-radiers qui appartiennent au même faciès d'écoulement.

\section{RÉSULTATS}

\section{Caractérisation de l'habitat sur les radiers étudiés}

Compte tenu des différences de débit d'une année à l'autre $\left(2,2 \mathrm{~m}^{3} \cdot \mathrm{s}^{-1}\right.$ en 1996 contre $1,2 \mathrm{~m}^{3} . \mathrm{s}^{1}$ en 1997), les caractéristiques de l'habitat avant enlèvement des végétaux diffèrent sensiblement entre 1996 et 1997 (Tableau I), le lit mouillé de la rivière étant peu affecté ( $-1 \%$ de surface en eau sur le radier amont et - $5 \%$ sur le radier aval en 1997). On enregistre entre 1996 et 1997 une baisse moyenne des profondeurs de $10 \mathrm{~cm}$ et une baisse moyenne des vitesses de $10 \mathrm{~cm} . \mathrm{s}^{-1}$. Cependant, les deux années les pourcentages de recouvrement en renoncules restent supérieurs à $70 \%$ et ils sont comparables sur les demi-radiers appartenant au même faciès (écart maximum de $10 \%$ entre la rive droite et la rive gauche sur le radier aval en 1997). Les biovolumes (mesurés en 1997 seulement) sont peu différents d'un demiradier à l'autre (35 et $43 \%$ à l'amont ; 39 et $44 \%$ à l'aval). D'un point de vue hydraulique, et indépendamment des différences annuelles de débit, les conditions d'écoulement sur le radier aval sont plus homogènes que sur le radier amont. En effet, sur ce dernier, les profondeurs et les vitesses moyennes mesurées en rive droite sont significativement plus élevées (test $t, p \leq 0,05$ ) qu'en rive gauche, alors qu'aucune différence n'est mise en évidence sur le radier aval. Pour ce qui concerne la granulométrie, les fractions dominantes sont les mêmes en 1996 sur chaque demi-radier, à l'amont comme à l'aval (blocs, pierres et sables grossiers). Les proportions de blocs et de pierres sont significativement plus fortes à l'amont en rive droite (test de Wilcoxon, $\mathrm{p} \leq 0,05$ ), confirmant l'hétérogénéité de ce radier.

\section{Impact de l'enlèvement des renoncules sur l'hydraulique (Figure 3)}

La courbe moyenne des DFx tracée à partir des 4 points $(1996,1997$, amont, aval) montre que le retrait des macrophytes sur les demi-radiers expérimentaux entraine une diminution des classes de profondeur supérieures à 30 ou $35 \mathrm{~cm}$ au profit des classes intermédiaires $(20$ à $30 \mathrm{~cm}$ ) sur l'ensemble du radier (Figure 3). Ces différences sont dans tous les cas significatives à $5 \%$ (test de Wilcoxon), sauf sur le radier amont en 1997 $(p \leq 0,10)$. En moyenne sur les deux radiers et toutes années confondues, l'enlèvement des renoncules a induit une baisse de $15 \%$ de la profondeur d'eau des radiers. Par contre, les conséquences de l'enlèvement des végétaux sur les vitesses de courant sont opposées sur les deux types d'habitat. Sur les demi-radiers expérimentaux, on observe une augmentation de fréquence des classes de vitesses fortes (comprises entre 40 et $70 \mathrm{~cm} . \mathrm{s}^{\prime}$ ) et une baisse de fréquence des classes de vitesses plus faibles $20-30 \mathrm{~cm} . \mathrm{s}^{-1}$ (et aussi $30-40 \mathrm{~cm} . \mathrm{s}^{-1}$ dans une moindre mesure). L'augmentation des vitesses de courant est alors en moyenne de $60 \%$ dans les demi-radiers expérimentaux, tendance significative quelles que soient la localisation (amont ou aval) et l'année d'étude (test de Wilcoxon, $p \leq 0,05$ ). Sur les demi-radiers intacts, 


\section{Tableau I}

Comparaisons des caractéristiques mésologiques des radiers (côtés droits et côtés gauches) avant enlèvement de la végétation aquatique. Profondeur et vitesse : valeurs moyennes et écarts types entre parenthèses (180 mesures sur le radier aval et 125 sur le radier amont chaque année). Granulométrie : les pourcentages de recouvrement ne sont donnés que pour les 3 classes les plus représentées (180 mesures sur le radier amont et 125 sur le radier aval chaque année). Code granulométrique utilisé (diamètre des particules en $\mathbf{m m}$ ) : $\mathbf{L i}=$ limon $(\leq 0,02) ; \mathrm{St}=$ sable fin $(0,02$ à 0,2$) ; \mathrm{Sg}=$ sable grossier $(0,2$ à 2$) ; \mathrm{Gr}=\operatorname{gravier}(2 \mathrm{à}$ 20) ; Ga $=$ galet $(21$ à 100); $\mathrm{Pi}=$ pierre $(101$ à 200) ; $\mathrm{BI}=$ bloc $(201$ à 500) et Ro $=$ rocher $(\geq 501)$. Macrophytes : pourcentages de recouvrement et de volume occupé (90 mesures sur le radier aval et 75 sur le radier amont chaque année). Valeurs en gras : différences significatives entre demi-radiers contigus (comparaison de moyennes, test $t, p<0,05$ ).

\section{Table I}

Comparisons between the physical characteristics of the left side and the right side of the riffles before aquatic weeds removal. Depth and water velocity : mean values and standard deviations in brackets (on average 180 points of measurement on downstream riffle and 125 on upstream riffle each year). Bottom substrate : \% of the surface (on average 180 points of measurement on downstream riffle and 125 on upstream riffle each year) only for the three most frequent classes in the habitat. Substratum particle size $(\mathrm{mm}): \mathrm{Li}=$ silt $(\mathbf{S} 0.02) ; \mathrm{Sf}=$ fine sand (0.021-0.2) ; $\mathrm{Sg}=$ coarse sand (0.21-2) $\mathrm{Gr}=$ gravel $(2.1-20) ; \mathrm{Ga}=$ pebble $(20.1-100) ; \mathrm{Pi}=$ cobble $(101-200) ; \mathrm{BI}=$ boulder $(201-500) ;$ Ro = large boulder $(z 501)$. Macrophyte cover and volume : \% of the surface and of the water volume (on average 90 points of measurement on downstream riffle and 75 on upstream riffle each year). Bold characters : significant differences between the mean habitat characteristics recorded on the left side and the right side for each riffle (mean value $t$-test, $p<0.05$ ).

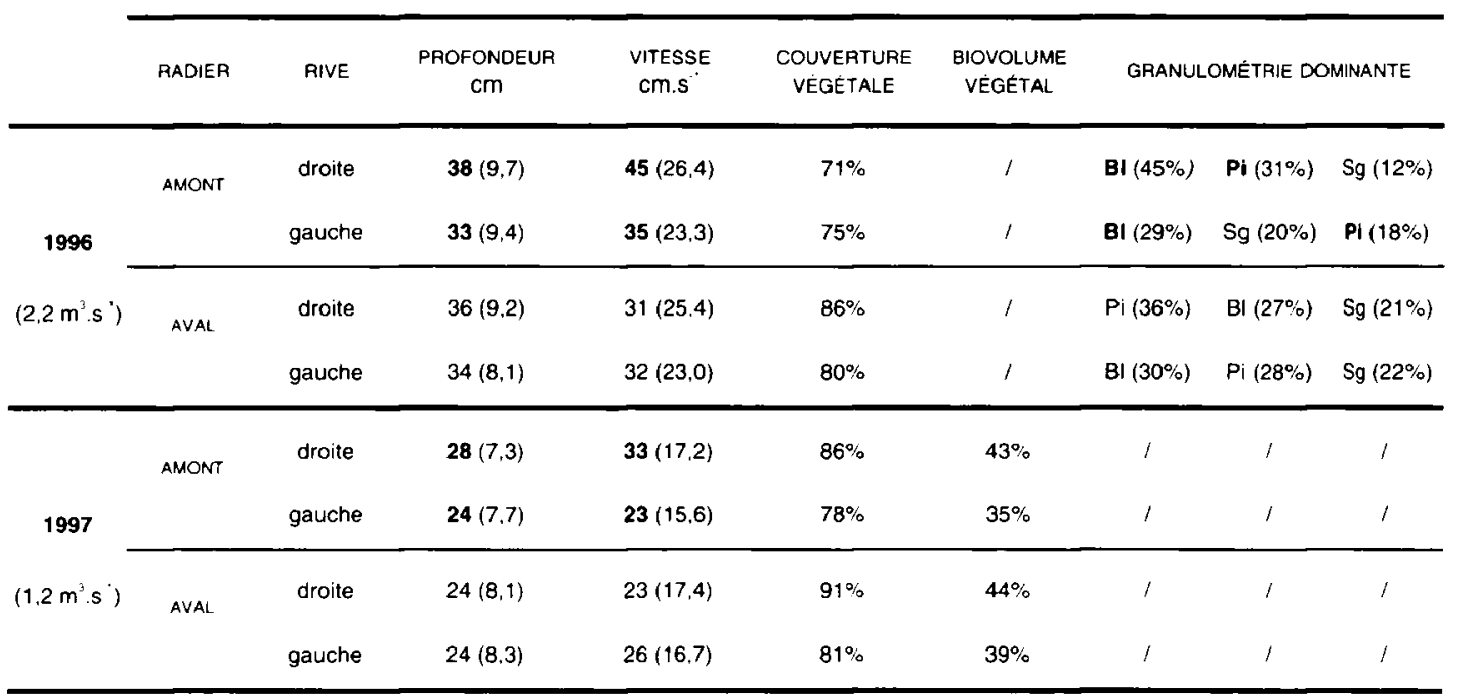

on observe une diminution sensible des classes de vitesses intermédiaires à fortes (comprises entre 30 et $70 \mathrm{~cm} . \mathrm{s}^{-}$) et une augmentation de fréquence des classes de vitesses faibles à moyennes (10 à $30 \mathrm{~cm} \cdot \mathrm{s}^{-1}$ ). Ces différences ne sont significatives (test de Wilcoxon, $p \leq 0,05$ ) qu'en 1997 sur le radier aval et en 1996 sur le radier amont. Globalement, le retrait des macrophytes sur les demi-radiers expérimentaux a induit une baisse de vitesse d'environ $25 \%$ dans les demi-radiers gardés intacts. Lorsque la granulométrie du substrat a été estimée (1996), les modifications enregistrées sur les fractions granulométriques ne sont jamais significatives, quelle que soit la localisation (test de Wilcoxon, $p>0,1$ ). 
Demi-radiers expérimentaux

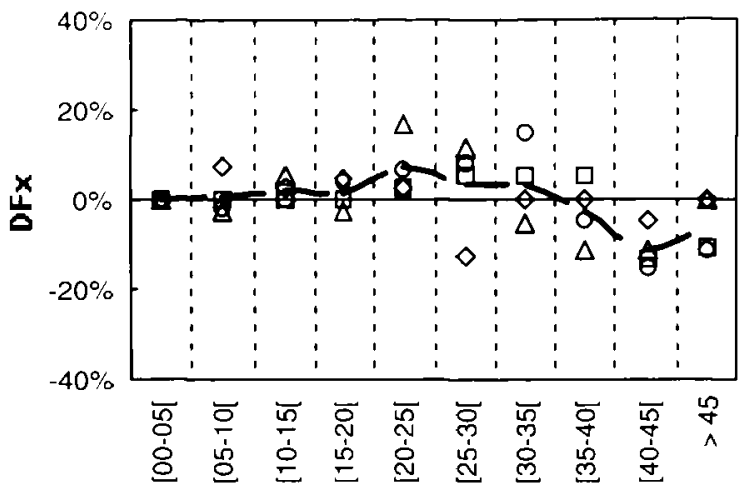

Classes de profondeur $(\mathrm{cm})$

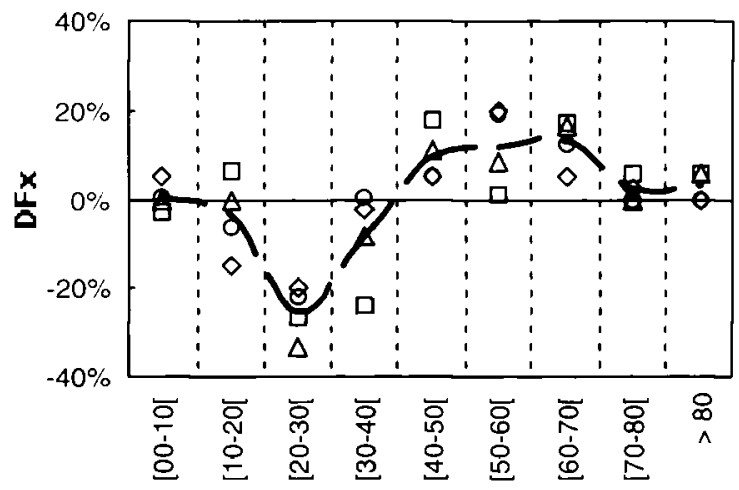

Classes de vitesse $\left(\mathrm{cm} \cdot \mathrm{s}^{-1}\right)$
Demi-radiers intacts

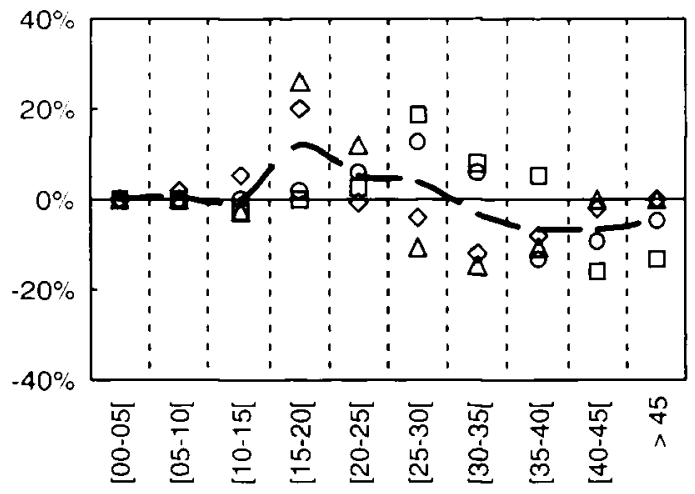

Classes de profondeur $(\mathrm{cm})$

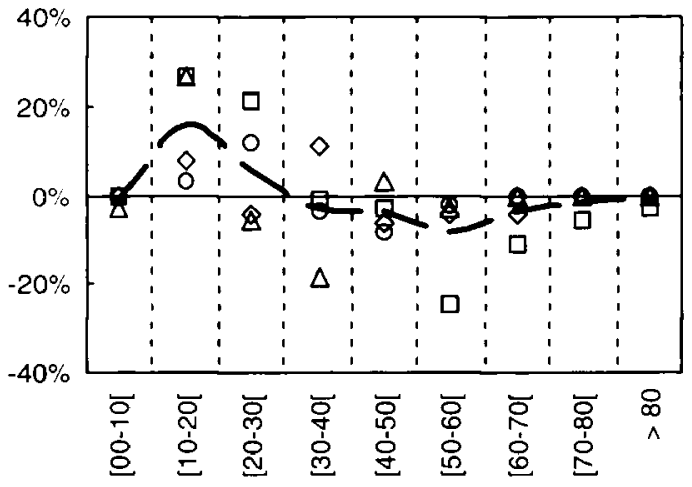

Classes de vitesse $\left(\mathrm{cm} \cdot \mathrm{s}^{-1}\right)$

\section{Figure 3}

Modifications des profondeurs et des vitesses de courant après enlèvement des renoncules sur les demi-radiers expérimentaux et intacts (90 mesures sur le radier aval et 75 sur le radier amont chaque année). Pour chaque classe d'habitat, $\mathrm{DF}_{\mathrm{x}}$ représente la différence de fréquence de la classe avant et après enlèvement des renoncules (voir Matériel et Méthodes pour plus de détails). Lorsque $\mathrm{DF}_{\mathrm{x}}<0$, la fréquence de la classe d'habitat considérée diminue après l'enlèvement des végétaux ; lorsque $D F_{x}>0$, la fréquence de la classe d'habitat considérée augmente après l'enlèvement des végétaux. Carrés : radier amont en 1996 ; ronds : radier aval en 1996 ; triangles : radier amont en 1997 ; losanges : radier aval en 1997. Pointillés : tendance moyenne pour les deux années d'étude.

\section{Figure 3}

Water depth and velocity changes after macrophyte removal (on average $\mathbf{9 0}$ points of measurement on downstream riffle and 75 on upstream riffle each year) - " expérimentaux " : macrophytes removed ; " intacts " : macrophytes left. For each habitat class (X-axis), $\mathrm{DF}_{\mathrm{x}}$ (Y-axis) is the difference between the frequencies of the class before and after macrophyte removal. When $D F_{x}<0$, the frequency of the class decreased after macrophyte removal ; when $D F_{x}>0$, the frequency of the class increased after macrophyte removal. Squares : upstream riffle in 1996 ; circles : downstream riffle in 1996 ; triangles : upstream riffle in 1997 ; diamonds : downstream riffle in 1997. Dotted lines : average trend for both years. 


\section{Communauté pisciaire des habitats avec et sans renoncules}

Un total de neuf espèces a été capturé au cours des deux années : juvénile de saumon atlantique (Salmo salar L.), truite commune (Salmo trutta L.), anguille (Anguilla anguilla L.), juvénile de lamproie marine (Petromizon marinus L.), lamproie de Planer (Lampetra planeriBloch), loche franche (Barbatula barbatula L.), vairon (Phoxinus phoxinus L.), chabot (Cottus gobio L.) et goujon (Gobio gobio L.). Le même nombre de taxons a été pêché dans les habitats avec et sans végétaux. Les biomasses totales de poissons pêchés en radier s'élèvent à $30,6 \mathrm{~kg} / \mathrm{ha}$ en 1996 et $38,9 \mathrm{~kg} / \mathrm{ha}$ en 1997 . En moyenne sur les deux années, $58 \%$ de la biomasse a été pêché dans les habitats végétalisés (61\% en 1996 et $55 \%$ en 1997). Pour les lamproies (lamproie marine et lamproie de Planer confondues), les truites et les juvéniles de saumon d'un an $\left(1^{+}\right)$, les effectifs ne représentent que $4 \%$ des captures totales et sont trop faibles pour estimer correctement les densités. Ces espèces ont donc été éliminées de l'analyse ultérieure. On note toutefois que toutes années et tous secteurs confondus, la moitié $(53 \%)$ des 45 truites capturées a été pêchée dans les habitats avec renoncules, alors que la majorité $(64 \%)$ des 22 saumons d'un an pêchés a été capturée dans les habitats sans renoncule.

En moyenne sur les deux campagnes de pêche, les densités en habitats végétalisés s'élèvent à 157 ind. $100 \mathrm{~m}^{2}$, contre seulement 93 ind.100 $\mathrm{m}^{-2}$ dans les habitats sans végétaux (Figure 4). Cette différence globale de densité s'accompagne de différences de composition du peuplement pisciaire entre les habitats avec et sans renoncules. Dans les habitats sans renoncule, les juvéniles de l'année de saumon atlantique $\left(0^{*}\right)$ sont numériquement dominants avec une densité moyenne de 30 ind. $100 \mathrm{~m}^{-2}$ et ils contribuent alors pour $32 \%$ à la totalité du peuplement pisciaire. La deuxième espèce est la loche avec 20 ind. $100 \mathrm{~m}^{2}$ en moyenne ( $21 \%$ du peuplement), l'anguille, le goujon, le vairon et le chabot n'étant représentés qu'à hauteur de 10 à 13 ind.100 $\mathrm{m}^{-2}$ en moyenne (11 à $14 \%$ du peuplement). Dans les habitats végétalisés, la loche et le vairon sont numériquement majoritaires (47 ind.100 $\mathrm{m}^{-2}$ en moyenne pour chaque espèce) et représentent à eux deux $60 \%$ des effectifs du peuplement. L'anguille est la troisième espèce la plus représentée dans ce type d'habitat avec une densité moyenne de 23 ind. $100 \mathrm{~m}^{-2}$ (15\% du peuplement), tandis que les juvéniles de saumon, les goujons et les chabots ont les densités moyennes les plus faibles (respectivement 15,13 et 11 ind.100 $\mathrm{m}^{2}$ ) et sont les espèces minoritaires du peuplement (7 à 10\%). L'examen de la répartition des effectifs par classe de taille indique que la structure d'âge de la population des différentes espèces varie peu d'une année à l'autre ou d'un habitat à l'autre.

\section{Variations des densités estimées par habitat et par année (Figure 5)}

Chabot et Goujon. Les densités de chabot sont relativement stables d'une année à l'autre (autour de 11 ind. $100 \mathrm{~m}^{2}$ ), alors que les densités de goujon passent de 6 ind. $100 \mathrm{~m}^{2}$ en moyenne en 1996 ( $A$ et B) à 17 ind. $100 \mathrm{~m}^{-2}$ en 1997 ( $C$ et $D$ ). Des espèces étudiées, les densités de chabot et de goujon semblent les moins influencées par le retrait des végétaux aquatiques (test de Wilcoxon, $p=0,46$ et $p=0,27$ respectivement pour le chabot et le goujon).

Anguille. La répartition des anguilles en radier semble être influencée par la présence des renoncules (test de Wilcoxon, $p=0,10$ ), ce qui se traduit par des abondances deux fois plus fortes dans les habitats végétalisés, sauf sur le radier aval en 1996 (B) où des densités identiques sont observées $\left(17\right.$ ind $\left.100 \mathrm{~m}^{-2}\right)$ entre les deux types d'habitat.

Loche et Vairon. On assiste à une forte augmentation de densité pour ces deux espèces entre 1996 ( $A$ et $B$ ) et 1997 (C et D), de 13 à 44 ind. $100 \mathrm{~m}^{-2}$ pour les vairons et de 14 à 51 ind. $100 \mathrm{~m}^{-2}$ pour les loches. De même que pour l'anguille, la répartition des loches et des vairons est influencée par le retrait des renoncules (test de Wilcoxon, $p=0,07$ ). 


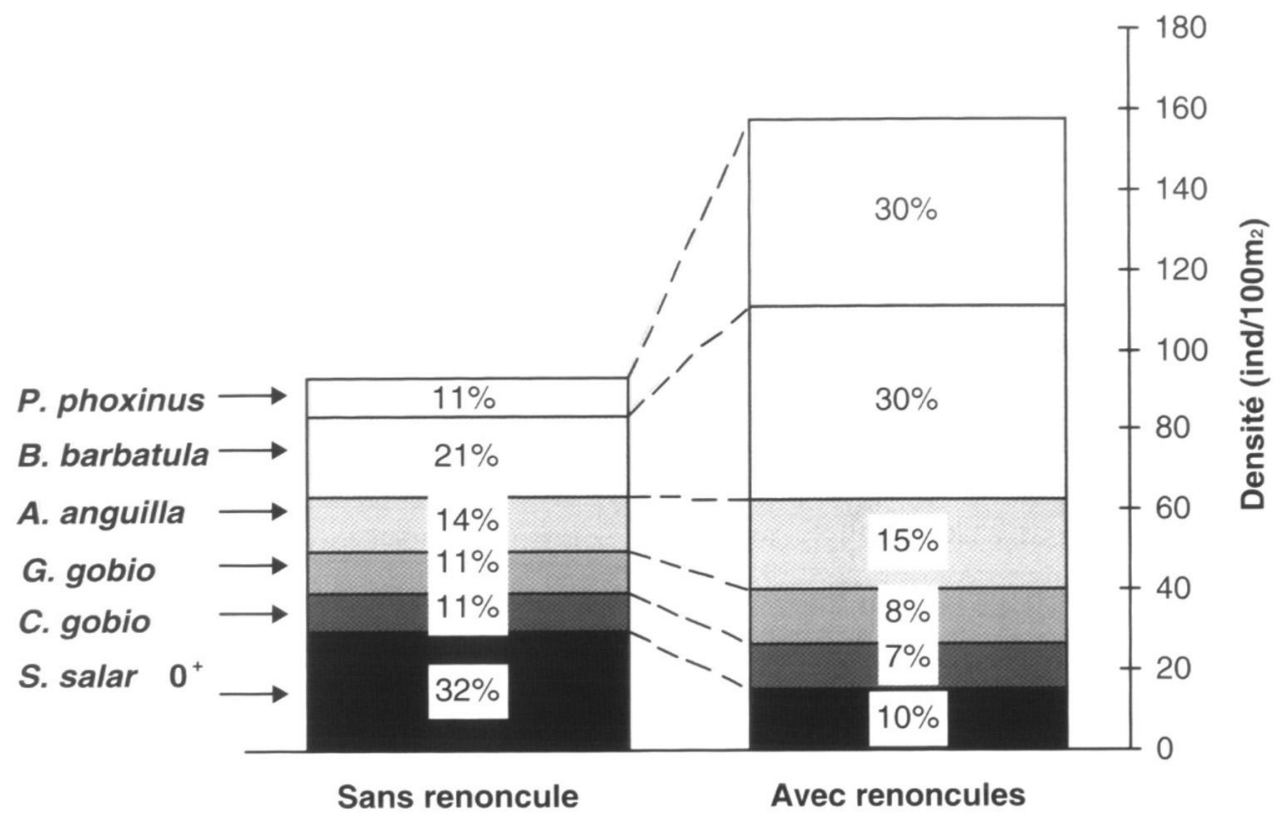

Figure 4

Composition du peuplement pisciaire dans les demi-radiers avec et sans renoncules. Pour chaque espèce, les densités correspondent aux valeurs moyennes pour les deux années d'étude, radiers amont et aval confondus. Les pourcentages indiqués représentent les contributions de chacune des espèces au peuplement pisciaire.

\section{Figure 4}

Fish community structure in habitats with macrophytes (right histobars) and without macrophytes (left histobars). Average densities (ind.100 $\mathrm{m}^{-2}$ ) are estimated from the two electrofishing surveys (1996 and 1997), upstream and downstream riffles pooled.

Les densités estimées dans les végétaux sont 2 à 3 fois supérieures pour la loche et 4 à 10 fois pour le vairon, à l'exception du secteur amont en 1996 (A) où peu de vairons ont été capturés.

Saumon 0+. En moyenne sur les deux radiers, la fréquentation de la zone d'étude par les juvéniles de saumon de l'année est identique en 1996 et en 1997 (23 ind.100 $\mathrm{m}^{2}$ ). Contrairement aux espèces précédentes, les densités de juvéniles de l'année sont 2 à 4 fois plus fortes dans les habitats sans végétaux (test de Wilcoxon, $p=0,07$ ), sauf en 1997 sur le radier amont $(C)$ lorsque la densité dans les végétaux est exceptionnellement forte (27 ind.100 $\mathrm{m}^{-2}$ contre environ 12 ind.100 $\mathrm{m}^{-2}$ en moyenne dans les autres habitats avec végétaux).

\section{DISCUSSION}

\section{Difficultés méthodologiques}

La présence de macrophytes dans le milieu rend techniquement difficile l'étude quantitative des populations de poissons. Dans les habitats lentiques et peu profonds (zone littorale de lac, bras mort de grand fleuve), il existe des méthodes d'échantillonnage par quadrats qui permettent de piéger les poissons dans des filets spéciaux (DEWEY et al., 
Salmo salar $0^{+}$

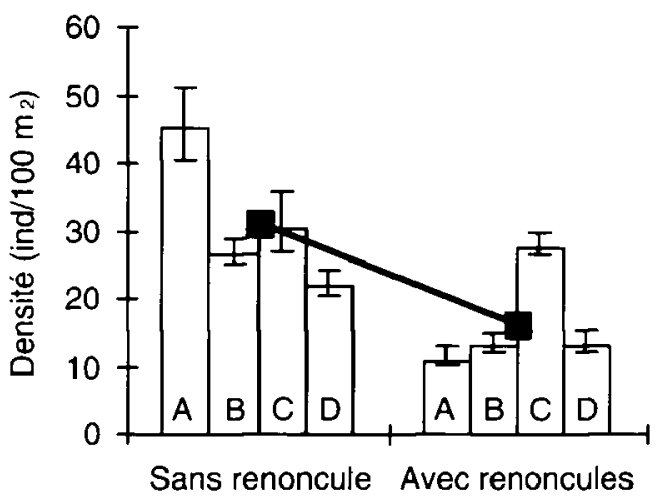

Phoxinus phoxinus

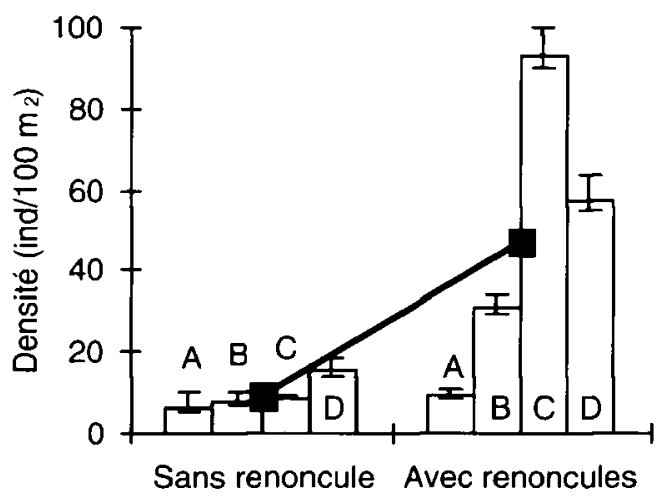

Gobio gobio

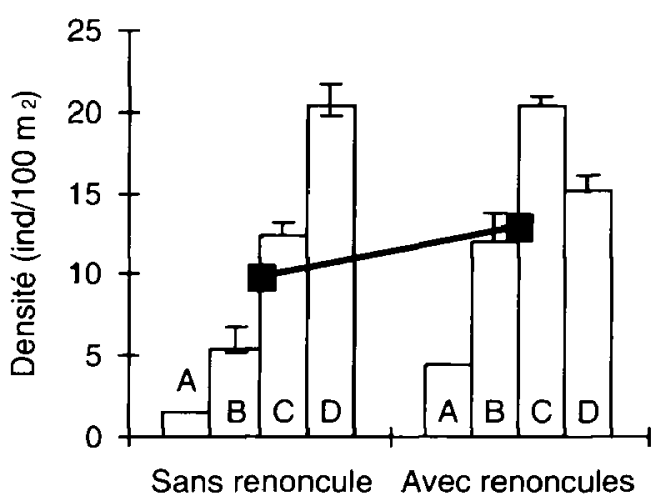

Anguilla anguilla

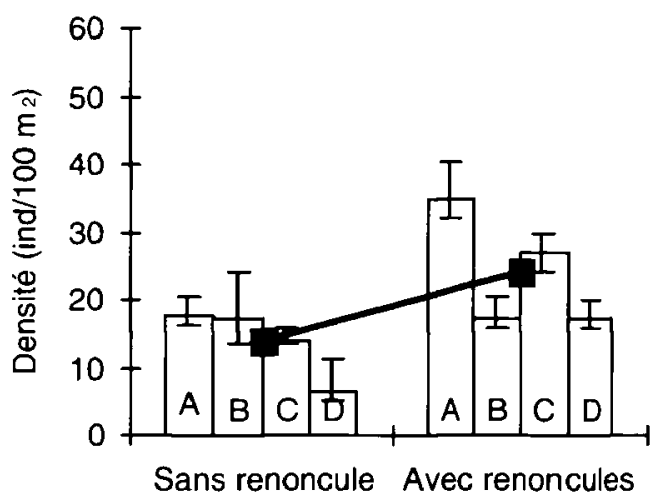

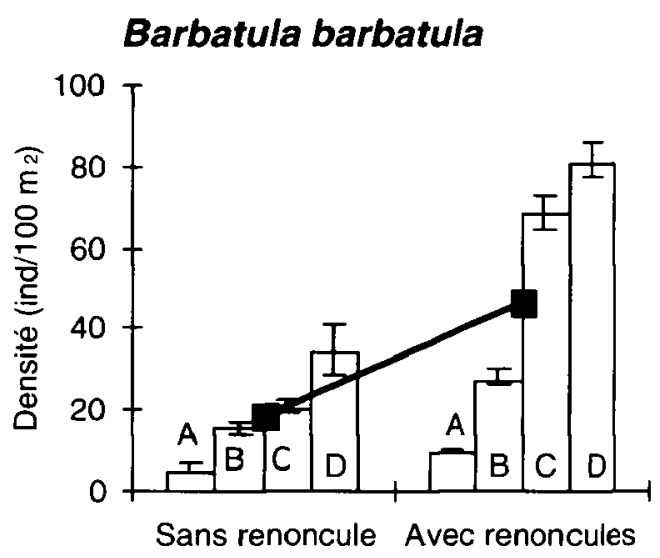

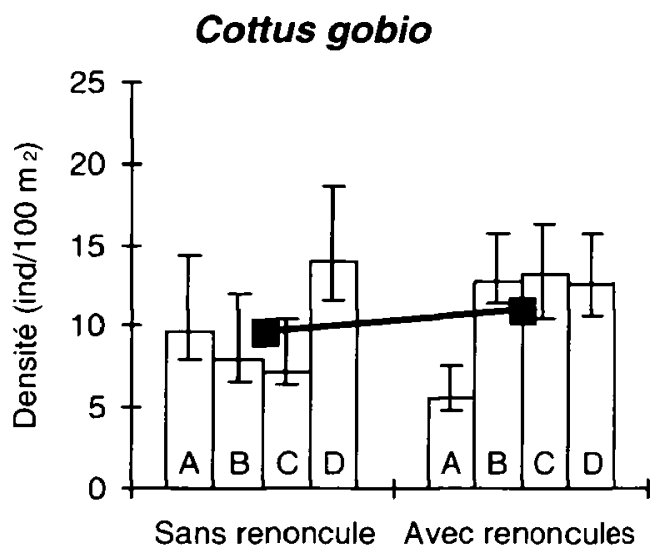

Figure 5

Estimation des densités de poissons en demi-radiers avec ou sans renoncules.

A : radier amont en 1996 ; B : radier aval en 1996 ; C : radier amont en 1997 ;

$D$ : radier aval en 1997. Carrés : valeurs moyennes. Traits verticaux : intervalles de confiance à $95 \%$ autour de l'estimation.

\section{Figure 5}

Estimated densities for each species in habitats with and without macrophytes. A : upstream riffle in 1996 ; $B$ : downstream riffle in $1996 ; C$ : upstream riffle in 1997 ; $D$ : downstream riffle in 1997 . Squares : mean values. Vertical lines : $95 \%$ contidence level for estimated populations. 
1989 ; DEWEY, 1992). Compte tenu de l'irrégularité des fonds dans un radier (cailloux, blocs) et des vitesses de courant élevées, une mise en place satisfaisante de filets s'est avérée impossible et l'échantillonnage par pêche électrique a donc été retenu pour l'inventaire des populations pisciaires. Les difficultés méthodologiques liées à cette technique sont connues. II s'agit dans un premier temps de prévenir les phénomènes d'émigration et d'immigration des poissons pendant la pêche, surtout en limite de secteur. Compte tenu de l'impossibilité d'assurer un isolement efficace des secteurs par la pose de filets, les secteurs ont été choisis de grande dimension ( 300 à $400 \mathrm{~m}^{2}$ chacun). De plus, un possible effet de bordure a été pris en compte grâce à la délimitation d'une bande de $4 \mathrm{~m}$ de large sur les limites entre les demi-radiers. La seconde difficulté réside dans la comparaison des densités estimées à partir de captures réalisées dans des milieux très différents, avec et sans renoncules. En effet, parmi les facteurs susceptibles de modifier l'efficacité de pêche (voir la synthèse proposée par ZALEWSKI et COWX, 1990), KARLSTRÖM (1976) remarque que la capturabilité des juvéniles de saumon peut être divisée par deux dans des habitats courants et végétalisés. Dans les radiers étudiés sur le Scorff, le tapis de renoncules couvre jusqu'à $70 \%$ de la surface, pouvant occuper $45 \%$ du volume d'eau disponible sur les radiers. Dans ces conditions, une partie des poissons reste inaccessible aux pêcheurs à cause de l'encombrement du lit de la rivière et, il y a donc un risque de sous-estimation des densités dans les végétaux par la méthode des passages successifs. Grâce à l'enlèvement des renoncules après les deux premiers passages de pêche, une évaluation plus juste des densités dans les secteurs végétalisés a été possible, permettant d'inclure dans l'estimation la fraction de la population piégée dans les renoncules. On ne peut toutefois écarter l'éventualité qu'une partie des poissons encore présente après les deux premiers passages ait fui pendant l'enlèvement des renoncules. C'est pour le saumon atlantique que la sous-estimation des densités qui en découlerait serait la plus gênante, car c'est la seule espèce pour laquelle les densités sont plus faibles en habitat végétalisé. Cependant, compte tenu du comportement territorial de l'espèce, elle est a priori peu susceptible d'avoir fui en dehors de la zone de pêche.

\section{Rôles de la végétation aquatique dans les habitats pisciaires}

Dans cette étude, les demi-radiers comparés étant en vis-à-vis, la diminution de la hauteur d'eau suite à l'enlèvement des renoncules s'est logiquement répercutée sur les deux types d'habitat (avec et sans renoncules). L'effet de l'enlèvement des macrophytes a surtout impliqué une augmentation des vitesses sur les habitats expérimentaux. Suite à l'augmentation du débit dans ces demi-radiers, les vitesses dans les habitats végétalisés ont diminué mais moins radicalement. On peut donc admettre qu'en termes d'habitat physique, ce sont surtout la vitesse de courant et la présence des macrophytes qui opposent les deux types d'habitat.

Les macrophytes représentent un facteur de l'habitat susceptible de jouer un rôle d'abri hydraulique pour les poissons. Les conditions hydrauliques sont en effet modifiées à l'échelle même du macrophyte. Par exemple, la vitesse de courant à $2 \mathrm{~cm}$ du fond peut être réduite 11 fois dans une touffe de callitriche par rapport à la vitesse à l'amont de ces touffes (SAND-JENSEN et MEBUS, 1996). Le rôle des macrophytes en tant qu'abri-cache est plus complexe. II peut s'agir tout d'abord de se dissimuler au regard des prédateurs : dans ce cas, un grand nombre de travaux, surtout en milieu stagnant, mettent en évidence le rôle de refuge joué par la végétation immergée (SAVINO et STEIN, 1982 ; GARNER, 1996 ; CHICK et McIVOR, 1997 ; DEWEY et al., 1997). Pour les poissons territoriaux, le macrophyte peut également faire office d'isolement visuel entre les congénères. Par exemple, FAUSCH (1993) a mis en évidence chez les juvéniles de truite (Oncorhynchus mykiss) en milieu naturel une plus grande utilisation des structures offrant un isolement visuel. Cependant, l'effet de l'isolement visuel sur la dimension des territoires et par conséquent sur la capacité d'accueil n'a pu être clairement identifié à notre connaissance. La présence des végétaux peut aussi influencer la capacité trophique du site, les macrophytes servant d'habitat pour 
un grand nombre de macroinvertébrés. Ainsi, WRIGHT (1992) a-t-il montré que la richesse taxonomique, l'abondance numérique et la biomasse des invertébrés étaient plus élevées dans trois types de macrophytes (dont les renoncules) que sur un substrat nu de sable et de gravier. Sur le Scorff, LAPCHIN (1976) remarque que les végétaux aquatiques des zones courantes peuvent héberger un nombre important de larves de diptères (essentiellement Chironomidae et Simulidae), l'effet positif des herbiers à renoncules sur le développement des larves de Simulidae ayant ensuite été mis en évidence sur la Nivelle (NEVEU et LAPCHIN, 1979). Cependant, les corrélations entre la biomasse des macrophytes et celle des invertébrés ne sont pas forcément directes (HUMPHRIES, 1996) et les apports trophiques pour le peuplement pisciaire restent à démontrer.

\section{Impact sur le peuplement}

Chaque radier étant divisé en deux types d'habitat, il est possible que des déplacements journaliers aient eu lieu entre les zones végétalisées et non-végétalisées, le poisson pouvant trouver un avantage à utiliser l'habitat avec renoncules seulement à un certain moment du nycthémère. Les données sur les changements nycthéméraux d'habitat sont peu nombreuses. Une étude sur la localisation diurne et nocturne de la loche, du chabot et du vairon a montré que pour ces espèces, il n'y avait pas de différences significatives dans l'occupation nycthémèrale des habitats de radier et de profond en ruisseau (ROUSSEL et BARDONNET, 1997). Le saumon $0^{+}$aurait une préférence pour des habitats moins courants la nuit, ceci à l'échelle du microhabitat (LE DREW et al., 1996). La taille importante des habitats (300 à $400 \mathrm{~m}^{2}$ ) diminue certainement la probabilité d'un déplacement nycthémèral entre les deux types d'habitat, mais elle ne l'exclue pas. Aussi, en l'absence de résultats sur les déplacements à cette échelle, les conclusions doivent être limitées à la localisation diurne des poissons.

Les taxons capturés dans les radiers du site d'étude correspondent aux espèces couramment rencontrées dans ce type d'habitat sur le cours principal du Scorff (BAGLINIĖRE, 1979). Si les radiers avec ou sans végétaux aquatiques abritent les mêmes espèces, les parts respectives des taxons sont différentes. Dans les habitats courants et sans renoncule, le peuplement est dominé par une espèce territoriale, le saumon, qui se déplace peu dans le milieu et qui est capable de se maintenir sans dépenses énergétiques dans des habitats à vitesse de courant élevée (PEAKE et al., 1997). Les densités atteignent 30 à 45 ind. $100 \mathrm{~m}^{-2}$ dans les zones sans renoncule, ce qui correspond aux densités les plus fortes observées ponctuellement sur le Scorff (BAGLINIĖRE et ARRIBE-MOUTOUNET, 1985 ; HAURY et BAGLINIÈRE, 1996). A l'inverse, les radiers fortement végétalisés sont numériquement dominés par la loche et le vairon, dont l'affinité pour la végétation aquatique et les conditions d'habitats qui lui sont liées (vitesses lentes, sédiments fins, abris) est connue (WELTON et al., 1983 ; MASTRORILLO et al., 1996 ; PRENDA et al., 1997 ; WATKINS et al., 1997). De plus, le vairon et la loche sont des espèces socialement plus tolérantes, s'alimentant selon un mode exploratoire, et les déplacements dans l'habitat peuvent être facilités à des vitesses de courant plus faibles. Ainsi, les espèces ayant une stratégie d'exploration de l'habitat pourraient se trouver avantagées par le développement des végétaux aquatiques.

Les préférences d'habitat des 0 . de saumons ont été abondamment décrites dans la littérature. Après l'émergence, les alevins se dispersent dans les habitats aux alentours des frayères et on les retrouve en grande majorité en été dans les faciès de type radier à substrat grossier (KARLSTRÖM, 1977 ; BAGLINIĖRE et CHAMPIGNEULE, 1982). A l'échelle du microhabitat, les profondeurs sélectionnées varient alors de 20 à $70 \mathrm{~cm}$, les vitesses focales de 10 à $60 \mathrm{~cm} . \mathrm{s}^{-1}$ (voir la synthèse proposée par HEGGENES, 1990). Dans les conditions de notre étude, les caractéristiques globales des faciès avant l'enlèvement des renoncules correspondent plutôt aux valeurs inférieures de ces gammes. La suppression des renoncules et l'accélération des vitesses de courant induites ont entraîné une 
redistribution des saumons $0^{*}$ en faveur des habitats non-végétalisés sauf dans un cas (radier amont 1997). La forte densité observée alors sur la zone avec renoncules est certainement à mettre en relation avec la structure de végétalisation sur ce demi-radier. En effet, la couverture macrophytique y était plus discontinue, laissant apparaître des veines de courant sans végétaux, mais trop étroites ( 1 à $1,5 \mathrm{~m}$ de large) pour être significatives sur les mesures de recouvrement réalisées. C'est dans ces veines de courant que des effectifs ponctuellement élevés en juvéniles de saumon ont été capturés en 1997. Ceci pourrait indiquer que, en deçà d'un effet macrophyte globalement négatif sur les densités de juvéniles lorsque les radiers sont fortement encombrés par les renoncules, des phénomènes de micro-distribution en veine de courant peuvent jouer un rôle compensatoire sur les densités moyennes dans certains radiers végétalisés.

\section{Implication pour la gestion}

Dans les conditions décrites précédemment, la pousse printanière des végétaux aquatiques est un facteur susceptible de créer temporairement des conditions d'habitat suboptimales pour les juvéniles de saumon atlantique. Pour cette espèce, l'estimation de la capacité d'accueil du bassin versant se traduit par sa capacité à produire des smolts (juvéniles de saumon migrant en mer) et elle est basée sur le calcul de la surface en habitat de production favorable pour les juvéniles (essentiellement radiers et rapides) accessible aux géniteurs. La modification temporaire de la qualité de ces habitats pourrait avoir un impact sur la croissance et la survie des juvéniles de saumon et donc sur la production en smoits. L'évaluation d'un tel impact demanderait des expérimentations à des échelles de temps et d'espace supérieures à ce qui a été réalisé ici. Cependant leur intérêt appliqué est certain, la gestion de la pêche à la ligne par TAC (Totaux Autorisés de Captures) étant basée sur le calcul de cette capacité d'accueil (PRÉVOST et PORCHER, 1996).

\section{REMERCIEMENTS}

Les auteurs remercient les relecteurs dont les remarques ont fortement contribué à l'élaboration de la version finale de cet article. Nos remerciements vont aussi à D. AZAM, N. JEANNOT, F. LEFEVRE, F. MARCHAND et aux nombreux étudiants ayant participé aux pêches électriques et aux mesures d'habitat, à $S$. AZAM pour les recherches bibliographiques sur informatique, ainsi qu'à J.Y. MOELO et à l'AAPPMA de Plouay (Morbihan) pour les facilités d'expérimentation et les autorisations de pêche électrique. Cette étude a été financée en partie par le Conseil Supérieur de la Pêche.

\section{BIBLIOGRAPHIE}

BAGLINIĖRE J.L., 1979. Les principales populations de poissons sur une rivière à salmonidés de Bretagne-Sud, le Scorff. Cybium, 3, 53-74.

BAGLINIĖRE J.L., CHAMPIGNEULLE A., 1982. Densité des populations de truite commune (Salmo trutta L.) et de juvéniles de saumon atlantique (Salmo salar L.) sur le cours principal du Scorff (Bretagne) : préférendum physiques et variations annuelles (1976-1980). Acta Oecologica, Oecol. Applic., 3, 241-256.

BAGLINIÈRE J.L., ARRIBE-MOUTOUNET D., 1985. Microrépartition des populations de truite commune (Salmo trutta), de juvéniles de saumon atlantique (Salmo salar) et des autres espèces présentes dans la partie haute du Scorff (Bretagne). Hydrobiologia, 120, 229-239.

BAGLINIÈRE J.L., CHAMPIGNEULLE A., 1986. Population estimates of juvenile Atlantic salmon (Salmo salar) as indices of smolt production in the River Scorff, Brittany. J. Fish Biol., 29, 467-482. 
BISSON P.A., MONTGOMERY D.R., 1996. Valley segments, stream reaches and channel units. In HAUER F.R., LAMBERTI G.A., Methods in stream ecology, Academic Press, London, 23-52.

BROWN A.V., BRUSSOCK P.P., 1991. Comparisons of benthic invertebrates between riffles and pools. Hydrobiologia, 220, 99-108.

CAILLEUX A., 1954. Limites dimensionnelles des noms des fractions granulométriques. Bull. Soc. Géol. Fr., 4, 643-646.

CARLE F.L., STRUB M.R., 1978. A new method for estimating population size from removal data. Biometrics, 34, 621-630.

CHAMPIGNEULLE A., 1978. Caractéristiques de l'habitat piscicole et de la population de juvéniles sauvages de saumon atlantique (Salmo salar L.) sur le cours principal du Scorff (Morbihan). Thèse 3ème cycle, Univ. Rennes, $92 \mathrm{p}$.

CHICK J.H., McIVOR C.C., 1997. Habitat selection by three littoral zone fishes : effects of predation pressure, plant density and macrophyte type. Ecol. Freshwat. Fish, 6, 27-35.

DANIEL H., HAURY J., 1995. Effects of fish farm pollution on phytocenoses in an acidic river (the Scorff river, South Brittany, France). Acta bot. Gallica, 142, 639-650.

DAWSON F.H., KERN-HANSEN U., 1979. The effect of natural and artificial shade on the macrophytes of lowland streams and the use of shade as a management technique. Int. Revue Ges. Hydrobiol., 20, 1451-1456.

DAWSON F.H., HASLAM S.M., 1983. The management of river vegetation with particular reference to shading effects of marginal vegetation. Landscape Planning, 10, 147-169.

DAWSON F.H., CHARLTON F.G., 1988. Bibliography on the hydraulic resistance or roughness of vegetated watercourses. Freshwater Biological Association, Occasional Publication $\mathrm{n}^{\circ} 25$, Dorset, UK. Freshwater Biological Association, River Laboratory, Wareham.

DEWEY M.R., HOLLAND-BARTELS L.E., ZIGLER S.J., 1989. Comparison of fish catches with buoyant pop nets and seines in vegetated and non vegetated habitats. North Am. J. Fish. Manage., 9, 249-253.

DEWEY M.R., 1992. Effectiveness of a drop net, a pop net, and an electrofishing frame for collecting quantitative samples of juvenile fishes in vegetation. North Am. J. Fish. Manage., 12, 808-813.

DEWEY M.R., RICHARDSON W.B., ZIGLER S.J., 1997. Patterns of foraging and distribution of bluegill sunfish in a Mississippi River backwater : influence of macrophytes and predation. Ecol. Freshwat. Fish, 6, 8-15.

FAUSCH K.D., 1993. Experimental analysis of microhabitat selection by juvenile steelhead (Oncorhynchus mykiss) and coho salmon ( $O$. kisutch) in a British Columbia stream. Can. J. Fish. Aquat. Sci., 50, 1198-1207.

GARNER P., 1996. Microhabitat use and diet of 0+ cyprinid fishes in a lentic, regulated reach of the river Great Ouse, England. J. Fish Biol., 48, 367-382.

HAURY J., BAGLINIĖRE J.L., 1996. Les macrophytes, facteur structurant de l'habitat piscicole en rivière à salmonidés. Etude de microrépartition sur un secteur végétalisé du Scorff (Bretagne-Sud). Cybium, 20, 111-127.

HEARNE J., JOHNSON I., ARMITAGE P., 1994. Determination of ecologically acceptable flows in rivers with seasonal changes in the density of macrophyte. Regul. Riv., 9, 177-184.

HEGGENES J., 1990. Habitat utilization and preferences in juvenile Atlantic salmon (Salmo salar) in streams. Regul. Riv., 5, 341-354.

HUMPHRIES P., 1996. Aquatic macrophytes, macroinvertebrate associations and water levels in a lowland Tasmanian river. Hydrobiologia, 321, 219-233.

KARLSTRÖM O., 1976. Quantitative methods in electrical fishings in Swedish salmon rivers. Zoon, 4, 53-63. 
KARLSTRÖM O., 1977. Habitat selection and population densities of salmon (Salmo salar L.) and trout (Salmo trutta L.) parr in Swedish rivers with some references to human activities. Act Univ. Upsaliensis, 404, $12 \mathrm{p}$.

LAPCHIN L., 1976. Méthodologie d'étude des invertébrés benthiques de deux ruisseaux à salmonidés de Bretagne. Thèse 3ème cycle, Univ. Rennes, $65 \mathrm{p}$.

LE DREW L.J., SCRUTON D.A., MCKINLEY R.S., POWER G., 1996. A comparison of habitat suitability indices developed from daytime versus nightime observations for Atlantic salmon in a regulated Newfoundland stream. In LECLERC M., CAPRA H., VALENTIN S., BOUDREAULT A., COTE Y. (eds), Proceedings 2nd International Symposium on Habitat Hydraulics, INRS-eau, Québec, vol. B, 33-44.

LOGAN P., BROOKER M.P., 1983. The macroinvertebrate faunas of riffles and pools. Water Res., 17, 263-270.

MASTRORILLO S., DAUBA F., BELAUD A., 1996. Utilisation des microhabitats par le vairon, le goujon et la loche franche dans trois rivières du sud-ouest de la France. Ann/s. Limnol., 32, 185-195.

NEVEU A., LAPCHIN L., 1979. Ecologie des principaux invertébrés filtreurs de la Basse-Nivelle (Pyrénées-Atlantiques). I. Simulidae (Diptera, Nematocera). Annls. Limnol., 14, 225-244.

PEAKE S., MCKINLEY R.S., SCRUTON D.A., 1997. Swimming performance of various freshwater Newfoundland salmonids relative to habitat selection and fishway design. J. Fish Biol., 51, 710-723.

PRENDA J., ARMITAGE P.D., GRAYSTON A., 1997. Habitat use by the fish assemblages of two chalk streams. J. Fish Biol., 51, 64-79.

PRÉVOST E., PORCHER J.P., 1996. Méthodologie d'élaboration des Totaux Autorisés de Captures (TAC) pour le saumon atlantique (Salmo salar L.) dans le Massif Armoricain. Propositions et recommandations scientifiques. GRISAM, Evaluation et gestion des stocks de poissons migrateurs. Doc. sci. tech., 1, $18 \mathrm{p}$.

ROUSSEL J.M., BARDONNET A., 1997. Diel and seasonal patterns of habitat use by fish in a natural salmonid brook : an approach to the functional role of the riffle-pool sequence. Bull. Fr. Pêche Piscic., 346, 573-588.

SAND-JENSEN K., JEPPESEN E., NIELSEN K., VAN DER BIJL L., HJERMIND L., NIELSEN L.W., IVERNEN T.M., 1989. Growth of macrophytes and ecosystem consequences in a lowland Danish stream. Freshwat. Biol., 22, 15-32.

SAND-JENSEN K., MEBUS J.R., 1996. Fins-scale patterns of water velocity within macrophyte patches in streams. Oikos, 76, 169-180.

SAVINO J.F., STEIN R.A., 1982. Predator-prey interaction between largemouth bass and bluegilis as influenced by simulated submersed vegetation. Trans. Am. Fish. Soc., 111, 255-266.

VAN DEVENTER J.S., PLATTS S., 1989. Microcomputer software system for generating population statistics from electrofishing data - user's guide for MicroFish 3.0. Gen. Tech. Rep. INT-254. Ogden, UT : U.S. Department of agriculture, Forest Service, Inter-mountain Research Station, $29 \mathrm{p}$.

VINSON M.R., VINSON D.K., ANGRADI T.R., 1992. Aquatic macrophytes and instream flow characteristics of a Rocky Mountain river. Rivers, 3, 260-265.

WATKINS M.S., DOHERTY S., COPP G.H., 1997. Microhabitat use by $0+$ and older fishes in a small English chalk stream. J. Fish Biol., 50, 1010-1024.

WELTON J.S., MILLS C.A., RENDEL E.L., 1983. Food and habitat partitioning in two small benthic fishes, Nemacheilus barbatulus L. and Cottus gobio L. Arch. Hydrobiol., 97, 434-454.

WRIGHT J.F., 1992. Spatial and temporal occurrence of invertebrates in a chalk stream, Berkshire, England. Hydrobiologia, 248, 11-30. 
ZALEWSKI M., COWX I.G., 1990. Factors affecting the efficiency of electric fishing. In COWX I.G., LAMARQUE P. (eds), Fishing with electricity, applications in freshwater fisheries management, Fishing New Books, Oxford, 89-111.

ZIPPIN C., 1956. An evaluation of the removal method of estimating animal populations. Biometrics, 12, 163-189. 